\title{
REALCE E INTEGRAÇÃO DE IMAGENS ORBITAIS ÓTICAS COM DADOS SRTM PARA MAPEAMENTO E ESTUDO DE GRANDES PLANÍCIES FLUVIAIS: EXEMPLOS DE APLICAÇÃO NO PANTANAL
}

\section{ENHANCEMENT AND INTEGRATION OF OPTICAL SATELLITE IMAGES WITH SRTM DATA FOR MAPPING AND STUDY OF LARGE FLUVIAL PLAINS: EXAMPLES IN THE BRAZILIAN PANTANAL}

Eder Renato Merino

Universidade Estadual Paulista - UNESP, Instituto de Geociências e Ciências Exatas - Campus de Rio Claro Av. 24A, 1515, Bela Vista, Rio Claro/SP, CEP: 13506-900, Brasil

Email: ermerino82@yahoo.com.br

\begin{abstract}
Fabiano do Nascimento Pupim
Universidade Estadual Paulista - UNESP, Instituto de Geociências e Ciências Exatas - Campus de Rio Claro Av. 24A, 1515, Bela Vista, Rio Claro/SP, CEP: 13506-900, Brasil Universidade de São Paulo - USP, Instituto de Geociências, Laboratório de Espectrometria Gama e Luminescência, Rua do Lago 562, São Paulo/SP, CEP: 05508-080, Brasil Email: fabianopupim@usp.com
\end{abstract}

Hudson de Azevedo Macedo Universidade Estadual Paulista - UNESP, Instituto de Geociências e Ciências Exatas - Campus de Rio Claro Av. 24A, 1515, Bela Vista, Rio Claro/SP, CEP: 13506-900, Brasil Email: hud_azevedo@hotmail.com

Mario Luis Assine Universidade Estadual Paulista - UNESP, Instituto de Geociências e Ciências Exatas - Campus de Rio Claro Av. 24A, 1515, Bela Vista, Rio Claro/SP, CEP: 13506-900, Brasil

Email: assine@rc.unesp.br

Informações sobre o Artigo

Data de Recebimento:

$10 / 11 / 2014$

Data de Aprovação:

$22 / 02 / 2015$

Palavras-chave:

SRTM; Pantanal; Sensoriamento Remoto; Sistemas Fluviais.

Keywords:

SRTM; Pantanal; Remote Sensing; Fluvial Systems

\section{Resumo:}

Estudos referentes à fisiografia e ao funcionamento de sistemas fluviais têm aumentado em todo mundo e o mesmo se aplica àqueles realizados nos grandes sistemas fluviais brasileiros. Inúmeras são as dificuldades para o estudo de grandes planícies fluviais destacando-se o acesso e a locomoção. A utilização de produtos de sensoriamento remoto e geotecnologias são ótimas alternativas para o estudo de áreas de difícil acesso. Com a crescente popularização das geotecnologias, os modelos digitais de elevação (MDEs) tornaram-se os produtos mais comuns para análise e extração de informações topográficas e os dados SRTM (Shuttle Radar Topography Mission) representam o mais completo MDE global. Entretanto, em áreas sazonalmente alagáveis e grandes planícies fluviais, muitas informações espaciais são omitidas devido à ausência de processamentos adequados do MDE. 
Este artigo apresenta técnicas de realce e integração de dados SRTM com imagens orbitais óticas para aplicação em estudos geomorfológicos em grandes planícies fluviais, tendo como exemplo as planícies do Pantanal. Após processados adequadamente, de acordo com a amplitude topográfica e as características morfológicas da área, dados SRTM ressaltaram a geometria de grandes sistemas fluviais distributários (megaleques) e suas formas deposicionais internas (canais com diques marginais e lobos distributários). A fusão IHS (Intensity-Hue-Saturation) de imagem composição R7G4B3 (Landsat 7 ETM+) e imagem de relevo sombreado derivado do SRTM TOPODATA (Hillshade) destacou o contato entre a planície do Pantanal e áreas do embasamento da bacia. Este trabalho demonstra que, mesmo em áreas extremamente planas como o Pantanal, processamentos adicionais melhoram significativamente a identificação de características morfológicas captadas por dados SRTM.

\begin{abstract}
:
Studies concerning the physiography and function of fluvial systems have increased both Worldwide and in the large Brazilian fluvial systems. These areas are difficult to study due to problems concerning accessibility and mobility. Remote sensing and geotechnologies are alternatives to partially overcome those difficulties. Digital elevation models (DEMs) have become the most common methods for analyzing and extracting topographic information and the SRTM (Shuttle Radar Topography Mission) provides the most complete global DEM. However, in seasonally flooded areas and large fluvial plains, much of the spatial information is omitted due to lack of adequate processing of the DEM. This paper presents techniques for the enhancement of SRTM data applied to geomorphological studies in large fluvial plains using the Brazilian Pantanal as an example. After being properly processed, according to the relief and morphological characteristics, SRTM data highlighted major distributary fluvial systems (megafans) and its internal characteristics (channel levees and distributary lobes). IHS fusion (Intensity-Hue-Saturation) of composition R7G4B3 (Landsat 7 ETM+) and shaded-relief image derived from SRTM TOPODATA (Hillshade) highlighted the boundary between the Pantanal and metamorphic rocks of the basement. This paper demonstrates that even in extremely flat areas like the Pantanal, additional processing improves significantly the identification of morphological characteristics captured by SRTM data.
\end{abstract}

\section{Introdução}

A ênfase da Geomorfologia no estudo das paisagens e processos formadores compartilha com outras disciplinas das Ciências da Terra a necessidade de compilar dados espaciais e então apresentá-los na forma de modelos ou mapas (GRIFFITHS et al., 2011). Mapas são compontes chave da pesquisa geomorfológica e a apresentação espacial e temporal de determinada feição oferece direcionamento ao fenômeno formador (VITEK et al., 1996). Ao longo dos anos a utilização de técnicas de Geoprocessamento e Sensoriamento Remoto tem sido uma ferramenta bastante eficaz na análise da paisagem e as suas relações entre formas, processos e escala (ex. BUTLE; WALSH, 1998; WALSH et al., 1998; BOCCO et al. 2001; SMITH; PAIN, 2009).

Dentre os vários ramos da Geomorfologia, os estudos sobre a fisiografia e funcionamento de sistemas fluviais têm recebido grande atenção nas últimas décadas, fato evidenciado pelo crescente número de publicações científicas sobre o tema em todo o mundo. Stott (2010;
2011) destacou que trabalhos sobre geomorfologia fluvial representaram aproximadamente $40 \%$ dos artigos publicados entre 2006 e 2009 no periódico Earth Surface Processes and Landforms (ESPL). Seguindo essa tendência e apesar de muitos desafios e dificuldades, os grandes sistemas fluviais brasileiros também têm sido objeto de diferentes investigações científicas, com destaque para os estudos sedimentológicos e geomorfológicos das bacias hidrográficas do Amazonas (LATRUBESSE; FRANZINELLI, 2005; LATRUBESSE et al., 2010, ROSSETTI, 2010; ROSSETTI et al., 2012), do Paraná ( STEVAUX, 1994; STEVAUX; SOUZA, 2004), do Araguaia (AQUINO et al., 2009; VALENTE; LATRUBESSE, 2012) e Paraguai no Pantanal (ASSINE; SOARES, 2004; ASSINE; SILVA, 2009; ASSINE et al., 2014).

As dificuldades de acesso e de locomoção são os fatores que mais se destacam no estudo das grandes planícies fluviais e áreas úmidas frequentemente alagáveis (wetlands). Devido a tais dificuldades e à monotonia topográfica, as formas de relevo da maioria 
das planícies fluviais carecem ainda de caracterização em detalhe. Neste contexto, o sensoriamento remoto propicia excelentes oportunidades para o estudo dessas áreas, pois fornece dados e informações sobre a superfície terrestre em grande variedade de escalas temporais e espaciais, e em diferentes resoluções radiométricas e espectrais (MATHER, 2010).

Nos últimos 15 anos, surgiram diversos programas para a difusão dos sistemas de informação geográfica (SIG) e políticas de distribuição gratuita de imagens orbitais por institutos governamentais nacionais (ex: INPE) e internacionais (ex: NASA) foram implementadas. Juntamente com a crescente popularização das geotecnologias, o uso de modelos digitais de elevação (MDEs) tornou-se comum para a extração de informações topográficas (CLARK, 1997; SMITH; CLARK, 2005; GONGA-SAHOLIARILIVA et al., 2011).

A Shuttle Radar Topography Mission (SRTM) foi uma missão conjunta das agências americanas NASA e National Geospatial-Intelligence em colaboração com as agências espaciais alemã e italiana. Os dados foram obtidos a bordo do ônibus espacial Endeavour e produziram o mais completo modelo digital de elevação global cobrindo cerca de $80 \%$ da superfície do planeta entre as latitudes $60^{\circ}$ norte e $56^{\circ}$ sul. A coleta dos dados altimétricos foi realizada por um sistema de radar de abertura sintética interferométrico (InSAR) operando nas bandas $\mathrm{C}$ e $\mathrm{X}$ com resolução espacial de $3 \operatorname{arcos}$ de segundos ( $\sim 90 \mathrm{~m})$ e 1 arco de segundo ( $\sim 30$ m) para algumas localizações. Após processamento, tais dados deram origem ao primeiro modelo digital de elevação global com alta resolução espacial (RABUS et al., 2003).

Produtos derivados do SRTM tornaram-se importantes fontes de dados em estudos geológicos e geomorfológicos, especialmente em áreas de difícil acesso e desprovidas de levantamentos topográficos sistemáticos (ex: BUBENZER; BOLTEN, 2008; HAYAKAWA et al., 2008; SYVITSKI et al., 2012; ZANI et al., 2012; ASSINE et al., 2014). Entretanto, em regiões com baixa amplitude topográfica, como em extensas planícies fluviais, muitas vezes, o potencial do SRTM é subestimado, acarretando em perda de informações preciosas para estudos do meio físico.

Estudos anteriores realizados na bacia sedimentar do Pantanal demonstraram que a combinação de técnicas de processamento de imagens ópticas e de radar, aliados à interpretação geomorfológica adequada, resultaram em grande ganho de informações espaciais (SILVA et al., 2007; ASSINE; SILVA, 2009; ZANI et al., 2009; KUERTEN; ASSINE, 2011; MERINO et al., 2013; ASSINE et al., 2014; MACEDO et al., 2014). Porém até o momento, tais técnicas não foram apresentadas de forma sistemática para que pudessem ser replicadas em outras regiões. Assim sendo, este artigo tem por objetivo apresentar técnicas de realce e integração de dados SRTM com imagens orbitais óticas, aplicadas a estudos e mapeamento geomorfológico de grandes planícies fluviais e áreas inundáveis (wetlands), utilizando-se como área piloto o Pantanal.

\section{Aspéctos Físicos Reginais da Área Piloto}

O Pantanal está localizado na Bacia do Alto Paraguai (BAP), abrangendo extensas áreas dos estados de Mato Grosso e de Mato Grosso do Sul, estendendo-se também por terras do Paraguai e da Bolívia (Figura 1). O Pantanal é considerado a maior área alagável (wetland) do planeta (JUNK et al., 2006), com extensão de aproximadamente $150.000 \mathrm{~km}^{2}$ de acordo com delimitação proposta por Padovani (2010) .

O clima é do tipo 'AW' de acordo com a classificação de Köppen, tendo inverno seco e verão úmido, com temperatura média anual em torno dos $25^{\circ} \mathrm{C}$ e precipitação média anual de 800 a $2000 \mathrm{~mm} /$ ano (CLARKE et al., 2003). A planície apresenta regime sazonal de cheias que causam a alagamentos periódicos na região, porém o pulso de inundação, ou onda de cheia (JUNK et al., 1989), e os diferentes domínios climáticos de seu entorno fazem com que diferentes áreas apresentem picos de inundação defasados. O período chuvoso inicia-se em outubro, com picos de precipitação em dezembro e janeiro, e período seco de junho a agosto.

Geologicamente, o Pantanal é uma ampla bacia sedimentar cenozóica controlada por falhas, com espessura sedimentar que ultrapassa os $500 \mathrm{~m}$ em seu depocentro. A origem da bacia tem sido relacionada à flexura litosférica do sistema de foreland adjacente aos Andes central (HORTON; DECELLES, 1997; USSAMI et al., 1999; CHASE et al., 2009). Diferentes sistemas deposicionais compõem a planície de sedimentação do Pantanal, onde se destaca a planície do rio Paraguai como coletora das águas de vários megaleques fluviais que drenam os planaltos adjacentes (ASSINE, 2003). Muitas feições morfológicas são formas reliquiares 
Merino E. R. et al.

(TRICART, 1982; AB'SABER, 1988; SOARES et al., 2003; ASSINE; SOARES, 2004), que testemunham uma evolução paleogeográfica condicionada por mudanças climáticas, incluindo variações do nível de base e da descarga fluvial, que vêm ocorrendo na área desde o Pleistoceno tardio (ASSINE, 2003; McGLUE et al., 2012; ASSINE et al., 2014).

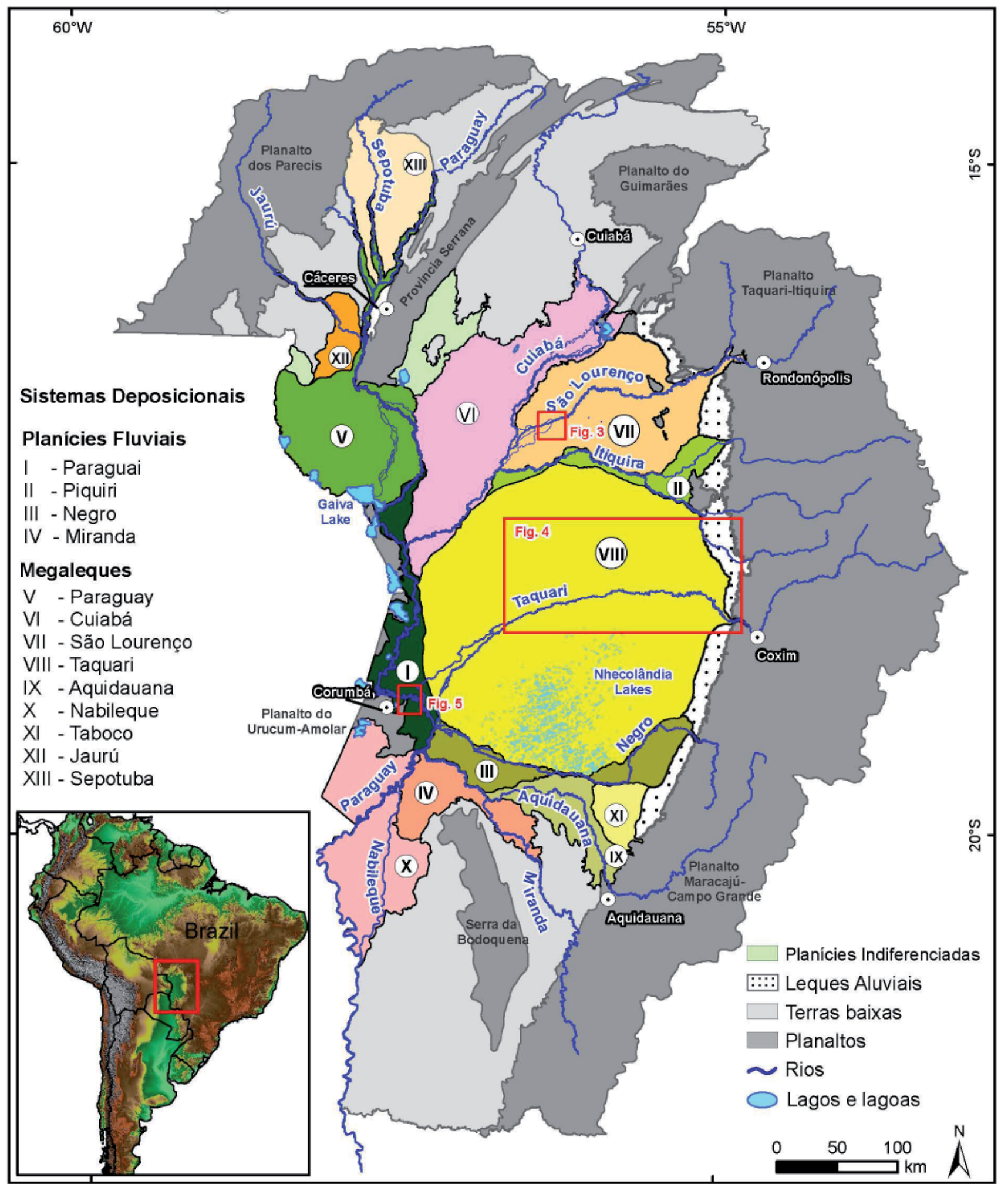

Figura 1 - Domínios geomorfológicos da BAP (Modificado de ASSINE; SILVA, 2009). Quadros vermelhos indicam localização das áreas utilizadas para teste dos processamentos. 


\section{Métodos e Procedimentos}

Tal como apresentado na caracterização regional, o Pantanal apresenta grande diversidade de sistemas e formas deposicionais com reduzida amplitude de relevo sendo um local ideal para testar a metodologia proposta. Tendo em vista tais características, distintas áreas com diferentes escalas de análise para aplicação dos métodos foram selecionadas.

Quatro tipos de dados orbitais foram utilizados para desenvolvimento do presente trabalho: 1) dados SRTM V.4 com 90m de resolução espacial, disponibilizados no sítio da CGIAR-CSI (http://srtm.csi.cgiar.org), que após reinterpolação e correção espacial dos dados (JARVIS, 2008) os disponibilizou em mosaicos regionais para todo planeta; 2) dados SRTM TOPODATA com $30 \mathrm{~m}$ de resolução espacial (VALERIANO; ROSSETTI, 2012 - www. dsr.inpe.br/topodata), disponibilizados para todo território brasileiro; 3) imagem Landsat 7 ETM + (cena 227/073 de 27/09/99) obtidas no site do Serviço Geológico Americano (http://glovis.usgs.gov), que disponibiliza grande acervo de imagens orbitais de diferentes sensores, com a grande vantagem de serem dados georreferenciados com acurácia geométrica e radiométrica; 4) mosaico GeoCover Circa 2000 (MDA FEDERAL, 2004), cenas S-21-15 e S-21-20 obtido junto ao Global Land Cover Facility (GLCF - http://glcfapp.glcf.umd.edu:8080/esdi), um centro de pesquisa com foco no uso de dados orbitais para estudos de mudanças no uso e cobertura do solo para sistemas locais e globais.

Os dados foram processados nos softwares Global Mapper (GLOBAL MAPPER LLC, 2009) para desenvolvimento dos mapas hipsométricos locais e regionais, ArcGis 9.3.1 (ESRI, 2009) para obtenção do relevo sombreado e fusão de imagens; ENVI 4.6 (ITT, 2008) para processamento digital das imagens Lansat $7 \mathrm{ETM}+$. Diferentes processamentos foram utilizados para realçar as informações espaciais dos dados SRTM e das imagens de satélite utilizadas na pesquisa, tais como: 1) customização dos intervalos altimétricos; 2) geração de relevo sombreado; 3) composição RGB; e 4) transformação IHS (Intensity-Hue-Saturation).

Para realçar as informações espaciais em áreas com baixa amplitude altimétrica são recomendados alguns procedimentos prévios: 1) análise geral das características geológicas e geomorfológicas da área por meio de imagens (óticas) e MDEs processados por métodos automáticos; 2) confecção de perfis topográficos para análise das quebras naturais de relevo e também da amplitude topográfica; 3 ) definição da escala de análise e intervalos altimétricos; 4) definição da paleta de cores para mapas hipsométricos. Recomenda-se, sempre que possível, a gradação de cores frias para cores quentes; quanto maior a escala de análise, menos tons de cores são recomendados para melhor realce das geoformas; 5) utilização de produtos derivados do SRTM, tais como o relevo sombreado, declividade, dentre outros; 6) utilização de imagens óticas para complemento da análise. Recomenda-se o uso de imagens do período seco quando os rios estão confinados em suas calhas naturais; e 7) fusão dos dados SRTM com imagens derivadas de processamentos digitais como transformação IHS, análise de principais componentes seletivas ou mesmo com uma simples composição RGB, são recomendados para regiões com poucas variações altimétricas.

\section{Resultados}

As altitutes topográficas do Pantanal variam entre 80 e 190 m acima do nível do mar, formando uma região plana circundada por planaltos e terrras baixas (“depressões”) adjacentes. A monotonia do relevo é rompida apenas por morros residuais distribuídos de forma esparsa na planície e também onde o contato planalto/planície ocorre de forma mais abrupta.

Na imagem GeoCover 1990 (Figura 2A) é possível observar os diferentes sistemas aluviais que compõem o Pantanal, com destaque para o megaleque do Taquari, grande forma circular no centro da imagem, e para a planície do rio Paraguai, área escura situada na porção oeste da bacia e mais sujeita às inundações periódicas. Todavia, ao utilizar dados SRTM com métodos automáticos de classificação hipsométrica os diferentes sistemas e feições são mascarados (Figura 2B). Com a aplicação da metodologia proposta o produto final apresenta qualidade bastante superior com sistemas e feições destacadas facilitando o mapeamento geomorfológico (Figura 2C).

Os resultados foram obtidos considerando-se as características topográficas e a amplitude altimétrica da planície. A customização dos intervalos foi de 8 a $15 \mathrm{~m}$ até a cota de $150 \mathrm{~m}$ e de $20 \mathrm{~m}$ da cota $150 \mathrm{~m}$ até a cota $205 \mathrm{~m}$ tendo sido utilizado intervalo único para os relevos adjacentes. A gradação variou de cores frias a quentes até a cota $150 \mathrm{~m}$ e cores frias entre as cotas 170 e $205 \mathrm{~m}$, com tons variando do branco ao cinza para que fosse destacado o contato entre a planície pantaneira e 
o embasamento cristalino. Para destacar os planaltos adjacentes, foram também utilizadas cores quentes em tons de vermelho, para que a cor fosse distribuída de forma homogênea visto que o objetivo central era destacar as formas de relevo da planície do Pantanal (Figura 2C). Após esse procedimento, a morfologia da planície foi ressaltada revelando formas semicirculares, com linhas de contorno topográfico concêntricas, que pertencem a diferentes sistemas fluviais.

O padrão geral das drenagens permitiu classificar boa parte dos sistemas como distributários, com rios principais cujos canais tiveram evolução marcada por avulsões sucessivas, como no caso do rio Taquari (ASSINE, 2005; ASSINE et al., 2005). A topografia da superfície apresenta perfis transversais convexos (Figura 2D) e longitudinais côncavos, geometria típica dos sistemas de leques deposicionais. Devido ao padrão distributário das drenagens, à grande área e ao gradiente topográfico baixo $(<0,5 \mathrm{~m} / \mathrm{km})$, tais sistemas são classificados como megaleques fluviais, conforme acepção de Horton; DeCelles (2001).
O perfil topográfico e o MDE customizado também destacaram que os principais megaleques provindos de leste (São Lourenço, Taquari e Aquidauana, Figura 1) são limitados por planícies fluviais denominadas interleques, tal como é o caso da planície do rio Negro (porção sul) entre os megaleques do Taquari e Aquidauana (MENDES et al., 2011), e a planície do rio Piquiri (porção norte) que separa os megaleques do São Lourenço e Taquari (PUPIM et al., 2014; Figura 2D). As planícies interleques (SINHA, 1996) são estreitas e caracterizadas pela presença de canais meandrantes em áreas bastante planas, com cotas topográficas inferiores às dos megaleques vizinhos, normalmente sujeitas a grandes inundações, e caracterizadas por constante abandono de canais e rupturas de meandros.

A utilização de tons de cinza (Figura 2C) entre as cotas altimétricas de 160 e 200 m evidenciou o limite entre a planície pantaneira e áreas mais elevadas nas porções leste e norte da área. Nas porções sul e oeste, devido a variações altimétricas, os limites entre a planície e áreas elevadas de entorno estão em altitudes mais baixas destacados pelas cores azul e verde claro.
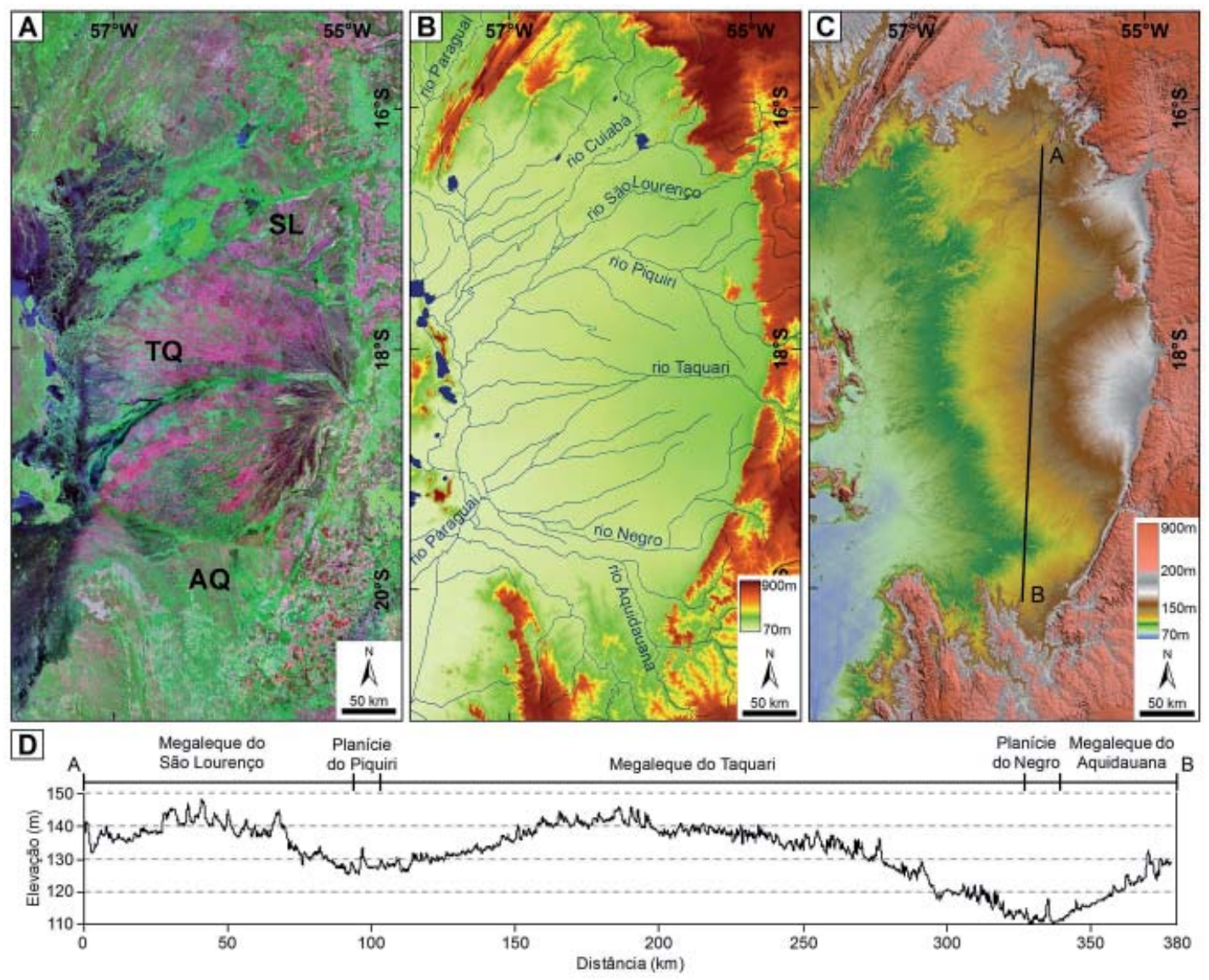

Figura 2 - Bacia do Alto Paraguai (BAP), cuja geomorfologia é caracterizada pela presença de sistemas de megaleques fluviais do Pantanal. A) Imagem Geocover; B) MDE com paleta automática e indicação de drenagem; C) MDE com ajuste na paleta de cores; D) Perfil Topográfico norte-sul na bacia do Pantanal. 
Avulsões seguidas por criação e abandono de canais são fenômenos comuns em regiões sujeitas a alagamentos periódicos. Dados SRTM aliados a sensores óticos (ex. Landsat) podem ser utilizados com bastante eficácia para destacar a ocorrência de tais fenômenos. Trechos que apresentam freqüente migração do canal e paleocanais preservados, presentes nos megaleques dos rios São Lourenço (Figura 3A-D) e Taquari (Figura 4), foram escolhidos para evidenciar a eficácia do método em destacar geoformas resultantes de tais processos.

No trecho selecionado do rio São Lourenço, tanto o canal principal quanto os paleocanais distributários apresentam morfologia de cordões compostos por canais e diques marginais (channel-levee ridges), que são caracterizados por contínua agradação vertical. A desaceleração lateral do fluxo durante transbordamentos faz com que os diques sejam construídos e a agradação no canal faz com que o rio corra progressivamente em níveis mais elevados que as planícies adjacentes
(ASSINE, 2005; ASSINE et al., 2014). Em imagens óticas essas feições apresentaram áreas homogêneas (Figura 3A) e pouca distinção entre as formas, porém, com a utilização de dados SRTM uma trama de canais distributários se sobrepondo (Figura 3B) foi realçada.

Por se tratar de uma área com extensão e geoformas reduzidas, bem como com baixa amplitude de relevo (100 a $155 \mathrm{~m}$ ) optou-se por utilizar poucas cores (porém contrastantes) na customização manual da paleta. Os intervalos altimétricos variaram entre 3 e $15 \mathrm{~m}$ e destacaram cordões de canais com diques marginais levemente mais elevados que a planície (Figura 3B e 3D), bem como paleocanais preservados (Figura 3D). Resultados similares foram obtidos na identificação de paleocanais na planície amazônica nas regiões dos rios Negro, Madeira e na Ilha de Marajó (ALMEIDAFILHO; MIRANDA, 2007; MANTELLI et al., 2009; HAYAKAWA et al., 2010).
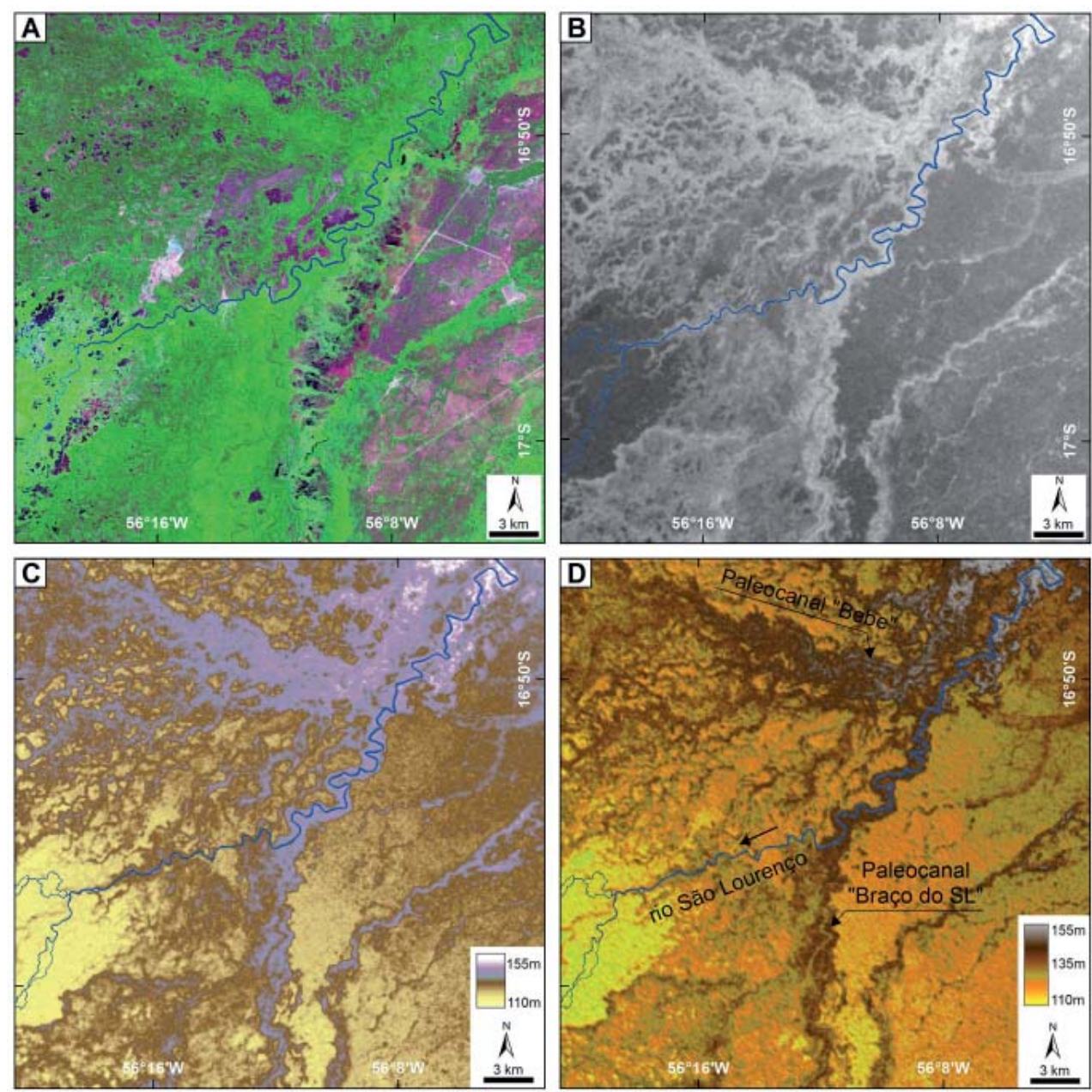

Figura 3 - Paleocanais distributários no lobo deposicional atual do rio São Lourenço. A) Imagem Geocover; B) MDE em tons de cinza; C) MDE customizado; D) MDE customizado. 
As imagens geradas a partir das técnicas propostas realçaram paleocanais distributários na região do megaleque do Taquari quando comparados MDEs gerados por interpoladores disponibilizados por softwares com MDEs customizados (Figura 4). Com este processamento a incisão do cinturão de meandros atual do rio
Taquari na entrada do Pantanal ficou melhor definida, além de destacar marcas de grandes avulsões a direita do cinturão atual. A diferença entre os MDEs é ainda maior nas áreas de contato entre planície pantaneira $\mathrm{e}$ os planaltos situados a leste.

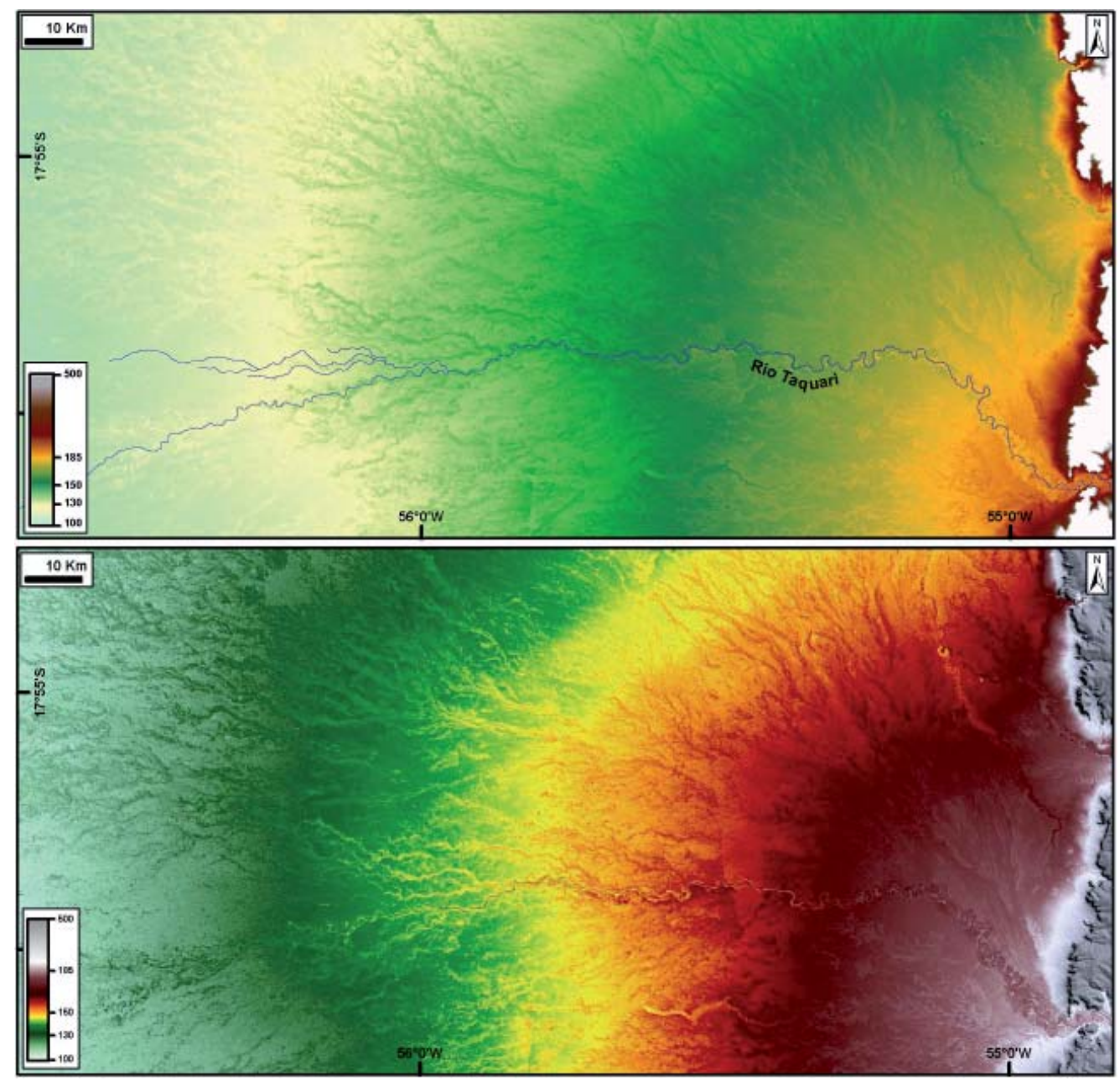

Figura 4 - Paleocanais distributários no megaleque do rio Taquari. A) MDE com paleta automática; B) MDE com ajuste nas paletas de cores.

A região escolhida na planície do rio Paraguai apresenta características geomorfológicas bastante contrastantes. Afloramentos do embasamento cristalino (no interior da bacia do Pantanal) e áreas elevadas do planalto do Urucum (na borda da bacia) contrastam com uma planície extremamente plana, repleta de canais e paleocanais com diques marginais com cotas altimétricas superiores às planícies adjacentes (Figura 5).

Para ressaltar as geoformas da planície, primeiramente, uma imagem dos valores de iluminação da superfície topográfica (hillshade ou relevo sombreado) com valores de pixel que variam de 0 (áreas sem ilumi- nação) a 255 (áreas totalmente iluminadas) com base na orientação do relevo (N-S para área) foi gerada. Melhores resultados são obtidos quando os parâmetros da fonte luminosa são definidos de forma perpendicular à orientação geral do relevo (azimute de $90^{\circ}$ e altitude de $25^{\circ}$ ). Devido à baixa amplitude topográfica da planície o exagero vertical do terreno foi de 3 vezes, destacando assim canais e seus diques marginais que na maioria dos casos não possuem elevações superiores a $1 \mathrm{~m}$.

Fusão entre a composição colorida R7G4B3 (Figura 4A) do sensor LandSat TM5 e o hillshade (Figura 4A), foi realizada pelo método da transformação 
IHS (Intensity-Hue-Saturation). A transformação IHS e a transformação de Brovey são os métodos de fusão de imagens de sensores remotos mais difundidos (CARPER et al., 1990; CHAVEZ et al., 1991; KOUTSIAS et al., 2000; TU et al., 2001). Normalmente é utilizada uma imagem pancromática de maior resolução espacial para realização do procedimento (MARTINS et al. 2009), todavia, no presente trabalho, optou-se por substituir a imagem ótica pela imagem de relevo sombreado do SRTM TOPODATA tal como procedimento adotado por Mendes et al. (2011).

Com base na imagem derivada da fusão IHS foi possível delimitar com mais precisão o limite entre a planície do rio Paraguai e os relevos residuais do embasamento cristalino. Pequenos morros, antes não visualizados na composição R7G4B3, foram realçados após a fusão das imagens. A imagem derivada da fusão IHS também realçou cordões de canais com diques marginais. $\mathrm{O}$ cinturão de meandros atual, bem como paleocinturões de meandros e paleocanais foram realçados. Macedo et al. (2014) utilizaram o mesmo processamento para a caracterização geomorfológica e definição do modelo evolutivo do trecho da planície do rio Paraguai entre as confluências dos rios Cuiabá e Miranda.

\section{Discussão}

Verstappen (1977), muito antes do Sensoriamento Remoto e seus produtos se tornarem amplamente difundidos, destacou que o resultado obtido da interpretação de dados orbitais depende diretamente dos métodos e produtos utilizados, mas principalmente da capacidade do intérprete em adotar procedimentos adequados para cada estudo. Desse modo, os resultados apresentados foram alcançados com base em conhecimentos prévios da geomorfologia do Pantanal, associados a técnicas de processamento digital de imagens, buscando-se extrair de cada área o maior número possível de informações.

Com a grande difusão das geotecnologias e distribuição gratuita de dados orbitais nos últimos anos (ex: imagens Landsat e SRTM), análises baseadas em dados de sensores remotos tornou-se atividade corriqueira em estudos e trabalhos ambientais e de planejamento (ex.VITEK et al., 1996; WALSH et al., 1998; SMITH; CLARK 2005; SIART et al., 2009; YANG et al., 2011). Modelagens hidrológicas e mapeamentos automáticos têm sido propostos utilizando-se produtos tais como o
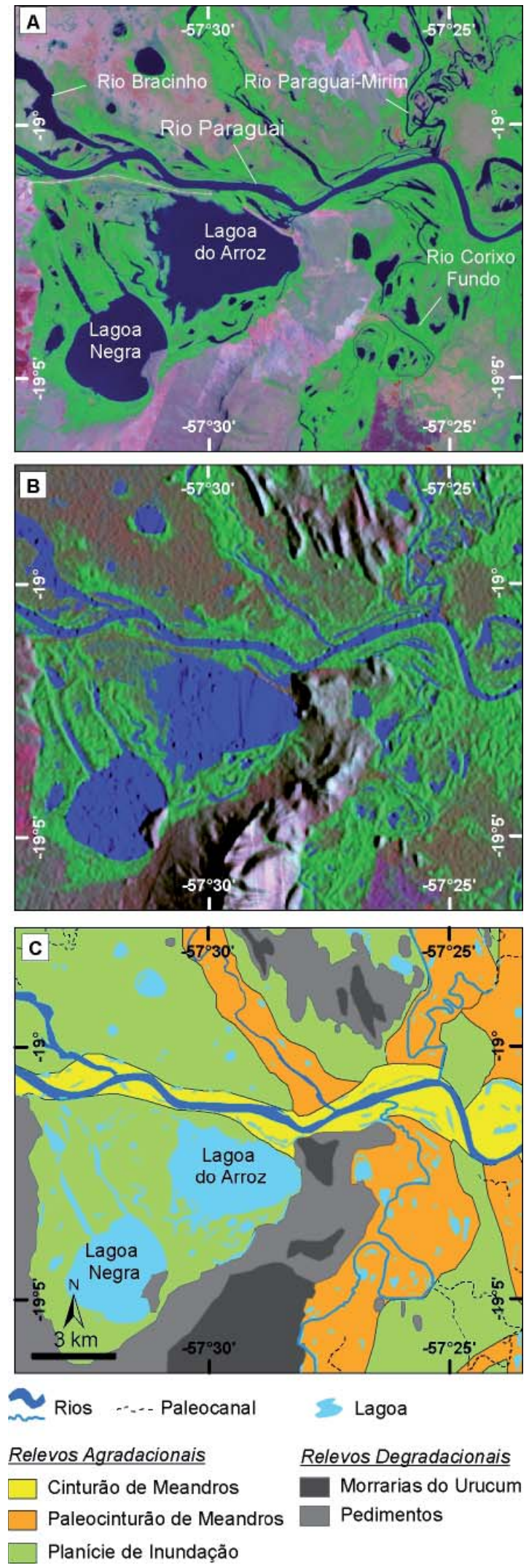

Figura 5 - Planície do rio Paraguai nas proximidades de CorumbáMS. A) Imagem Landsat 7 ETM+ R7G4B3; B) Hillshade fundido com a imagem ETM+; C) Interpretação geomorfológica (baseada em MACEDO et al., 2014). 
SRTM (DRĂGUŢ; BLASCHKE, 2006; SAADAT et al., 2008; PAZ et al., 2011; TINOS et al., 2014). Diversos pesquisadores do campo das geociências também têm utilizado dados SRTM em estudos sobre a evolução da paisagem (BLUMBERG, 2006; NAPIERALSKI et al., 2007; ROSSETTI; VALERIANO, 2007; BUBENZER; BOLTEN, 2008; SIART et al., 2008).

Zani et al. (2009) destacaram que MDEs constituem excelente ferramenta para reconhecimento de relações espaciais e mapeamento de formas deposicionais em ambientes com baixa declividade, como nos megaleques fluviais onde a superposição e o truncamento de canais distributários, bem como a progradação de lobos deposicionais, são constantes. Rossetti e Valeriano (2007) mostraram que o uso do MDE-SRTM processado de maneira adequada é altamente eficiente para destacar paleocanais e paleorredes de drenagens na Ilha de Marajó. Almeida-Filho e Miranda (2007) destacaram que dados SRTM possibilitaram revelar a existência de um antigo sistema de drenagem oculto pela floresta tropical na Amazônia Central, o qual não era visível tanto em imagens óticas como em imagens de radar.

Com base em dados altimétricos SRTM, Syvitski et al. (2012) estudaram diferentes planícies fluviais espalhadas pelo mundo. Elementos morfológicos de menor dimensão, típicos de sistemas fluviais e deltaicos, incluindo meandros ativos, canais de rios entrelaçados, barras fluviais, canais abandonados, espiras de meandro, lagoas de meandro abandonado, diques-marginais e crevasses splays foram destacados em imagens elaboradas com intervalos de cores de um metro, em conjuntos repetidos de $10 \mathrm{em} 10 \mathrm{~m}$. O mesmo procedimento poderia ser aplicado à planície do Pantanal e, provavelmente, contribuiria para o destaque de formas deposicionais com baixa variação altimétrica. No entanto, a repetição das cores em diferentes níveis altimétricos, prejudica a visualização das classes hipsométricas, bem como dificulta o reconhecimento de unidades de relevo em escala regional.

Trabalhos anteriores chamaram atenção para o fato de a banda C do SRTM interagir com componentes do dossel das arvores, criando "falsos" relevos e induzindo a erros de mapeamento. Dessa forma, os valores de elevações do SRTM referem-se à combinação da morfologia do terreno com a altura da cobertura vegetal (ex., VALERIANO et al., 2006; HAYAKAWA et al., 2010; LALONDE et al., 2010). Por outro lado, formações vegetacionais são intimamente relacionadas a processos fluviais (OSTERKAMP; HUPP, 2010), bem como, indicam como variações climáticas ou mudanças no uso do solo afetam paisagens e geoformas (KIRKBY, 1995). No Pantanal as florestas mesófilas (savanas) coincidem com áreas mais elevadas, não alagadas e com solos bem drenados (PRANCE; SCHALLER, 1982; POTT; POTT, 2004), tais áreas são representadas por maiores elevações no MDE (ZANI et al., 2012). Em estudo realizado na Amazônia, Mantelli et al. (2009) e Hayakawa et al. (2010) utilizaram dados SRTM para reconhecimento de paleomorfologias e, mesmo com a densa cobertura vegetal e a influência do dossel, tiveram sucesso na identificação de paleocanais e redes de drenagem ocultas pela vegetação, pouco evidentes em imagens geradas a partir de dados óticos.

O resultado final da customização do SRTM realizado neste trabalho (Figura 2C) corrobora a concepção de que o Pantanal não é uma planície geomorfologicamente homogênea, resultando em "diversos pantanais", cada um com características próprias de morfologia e dinâmica de inundações (ASSINE, 2003). Geoformas deposicionais e erosivas, que compõem a paisagem pantaneira, foram realçadas com a análise conjunta de dados SRTM customizados manualmente, com processamentos digitais de imagens relativamente simples de serem aplicados. Em síntese, os resultados apresentados neste trabalho detalham e consolidam a utilização de técnicas já parcialmente utilizadas com sucesso em trabalhos anteriores (ASSINE; SILVA, 2009; ZANI et al., 2009; KUERTEN; ASSINE, 2011; MERINO et al., 2013; ASSINE et al., 2014; MACEDO et al., 2014).

\section{Conclusão}

A utilização de técnicas de Sensoriamento Remoto em estudos geomorfológicos e geológicos não é novidade. No entanto, processamentos adicionais simples podem melhorar significativamente as características morfológicas captadas pelos dados da banda C do SRTM quando integrados com imagens orbitais óticas. A customização dos MDEs realçaram características morfológicas que facilitam a identificação e caracterização de megaleques fluviais e de planícies fluviais tributárias, possibilitando o desenvolvimento de estudos que buscam compreender melhor as características geomorfológicas e a evolução de planícies fluviais como a do Pantanal. O uso de 
geotecnologias e dados orbitais não elimina, entretanto, a necessidade da experiência de campo, principalmente para geocientistas. $\mathrm{O}$ conhecimento das formas e processos que modelam a evolução da paisagem estudada também é primordial para obtenção de bons resultados.

Os MDEs produzidos com intervalos altimétricos e paletas de cores definidas pelo usuário possibilitaram melhor visualização das características gerais de planícies aluviais. No caso da planície pantaneira, permitiram definir com mais precisão formas deposicionais dos megaleques fluviais com proveniência dos planaltos situados a leste, bem como de planícies sujeitas a grandes alagamentos nas porções sul e nordeste da bacia. Em escala local, ressaltou antigos canais abandonados que apresentam pouca expressão em dados óticos, bem como cordões formados por paleocanais margeados por paleodiques marginais.

Com base nos resultados obtidos, recomenda-se que, para obtenção de bons produtos com o uso do MDE/SRTM aplicado às planícies fluviais ou outras áreas com baixa declividade, não basta apenas aplicar paletas de cores pré-definidas nos softwares de SIG disponíveis ou paletas clássicas utilizadas para caracterização hipsométrica em áreas planálticas ou montanhosas. Os intervalos altimétricos e a disposição das cores devem ser ambos trabalhados de acordo com a amplitude topográfica e as características morfológicas da área. A fusão de MDEs com imagens óticas tem especial valor na identificação e caracterização de formas deposicionais de pequena expressão altimétrica, auxiliando em mapeamentos de detalhe e definição de áreas para levantamentos de campo.

\section{Agradecimentos}

Os autores agradecem à FAPESP (processo 2014/06889-2) e ao CNPq (processo 443437/2014-9) pelo apoio à pesquisa; ao CNPq pela bolsa $\mathrm{PQ}$ concedida a MLA (308563/2013-1), pelas bolsas de doutorado concedidas a ERM, FNP e HAM.

\section{Referências Bibliográficas}

AB'SÁBER, A. N. O Pantanal Mato-Grossense e a teoria dos refúgios. Revista Brasileira de Geografia, v.50, p. 9-57, 1988.

ALMEIDA-FILHO,R., MIRANDA, F.P. Mega capture of the Rio
Negro and formation of the Anavilhanas Archipelago, Central Amazônia, Brazil: Evidences in an SRTM digital elevation model. Remote Sensing of Environment, v. 110, p. 387-392, 2007.

AQUINO, S.; LATRUBESSE, E. M.; BAYER, MAXIMILIANO. Assessment of wash load transport in the Araguaia River (Aruana gauge station), Central Brazil. Latin American Journal of Sedimentology and Basin Analysis, v.16, p.119-128, 2009.

ANA/GEF/PNUMA/OEA. Programa de Ações Estratégicas para o Gerenciamento Integrado do Pantanal e Bacia do Alto Paraguai. Relatório Final/Agência Nacional de Águas -ANA. Brasília, 2004.

ASSINE, M.L. Sedimentação na Bacia do Pantanal MatoGrossense, Centro-Oeste do Brasil. Rio Claro, Universidade Estadual Paulista, Instituto de Geociências e Ciências Exatas, Tese de Livre-Docência, 106p, 2003.

ASSINE, M.L.; SOARES, P. C. Quaternary of the Pantanal, westcentral Brazil. Quaternary International, v.114, p. 23-34, 2004.

ASSINE, M.L., River avulsions on the Taquari megafan, Pantanal wetland, Brazil. Geomorphology, v. 70, p. 357-371, 2005.

ASSINE, M.L.; Padovani, C.R.; Zacharias, A.A.; Angulo, R.J.; Souza, M.C. Compartimentação geomorfológica, processos de avulsão fluvial e mudanças de curso do Rio Taquari, Pantanal Mato-Grossense. Revista Brasileira de Geomorfologia, v. 6, n. 1, p. 97-108, 2005

ASSINE, M.L.; SILVA, A. Contrasting fluvial styles of the Paraguay River in the northwestern border of the Pantanal wetland, Brazil. Geomorphology, v. 113 p. 189-199, 2009

ASSINE, M. L.; CORRADINI, F.A.; PUPIM, F.N.; McGLUE, M.M. 2014. Channel arrangements and depositional styles in the São Lourenço fluvial megafan, Brazilian Pantanal wetland. Sedimentary Geology, v. 301, n. 0, p. 172-184, 2014.

BLUMBERG, D. G. Analysis of large aeolian (wind-blown) bedforms using the Shuttle Radar Topography Mission (SRTM) digital elevation data. Remote Sensing of Environment, v. 100, n. 2, p. 179-189, 2006.

BOCCO, G.; MENDOZA, M.; VELÁZQUEZ,A. Remote sensing and GIS-based regional geomorphological mapping - a tool for land use planning in developing countries. Geomorphology, v. 39, n. 3-4, p. 211-219, 2001.

BUBENZER, O.; BOLTEN, A. The use of new elevation data (SRTM/ASTER) for the detection and morphometric quantification of Pleistocene megadunes (draa) in the eastern 
Sahara and the southern Namib. Geomorphology, v. 102, p. 221-231, 2008.

BUTLE, D. R.; WALSH, S. J. The application of remote sensing and geographic information systems in the study of geomorphology: An introduction. Geomorphology, v. 21, n. 3-4, p. 179-181, 1998.

CARPER, W. J.; LILLESAND, T. M.; KIEFER, R. W. The use of intensity-hue-saturation transformations for merging SPOT panchromatic and multispectral image data. Photogrammetric Engineering and Remote Sensing, v. 56, p. 459-67, 1990.

CHASE, C.G., SUSSMAN, A.J., COBLENTZ, D.D. Curved Andes: geoid, forebulge, and flexure. Lithosphere, v.1, n.6, p. 358-363, 2009

CHAVEZ, P.S, SIDE, S.C, ANDERSON, J.A. Comparison of Three different methods to merge multiresolution and multispectral data: TM and Spot Panchromatic. Photogrammetric Engineering \& Remote Sensing, v.57, n.3, p. 295-303, 1991.

CLARK, C. D. Reconstructing the evolutionary dynamics of former ice sheets using multi-temporal evidence, remote sensing and GIS. Quaternary Science Reviews, v. 16, n. 9, p. 10671092, 1997.

CLARKE, R. T. et al. Variabilidade temporal no regime hidrológico da bacia do rio Paraguai. Revista Brasileira de Recursos Hídricos, v.8, n.1, p. 201-211, 2003.

DRĂGUȚ, L.; BLASCHKE, T. Automated classification of landform elements using object-based image analysis. Geomorphology, v. 81, n. 3-4, p. 330-344, 2006.

ESRI - Environmental Systems Research Institue. 2009. ArcGis 9.3.1. Redlands - California.

GRIFFITHS, J. S.; SMITH, M. J.; PARON, P. Chapter One Introduction to Applied Geomorphological Mapping. In: MIKE J. SMITH, P. P. e JAMES, S. G. (Ed.). Developments in Earth Surface Processes: Elsevier, v. 15, 612p, 2011.

GLOBAL MAPPER LLC. Global Mapper Version 9.0 Software. Colorado, Parker, 2009.

GONGA-SAHOLIARILIVA, N.; GUNNELL, Y.; PETIT, C.; MERING, C. Techniques for quantifying the accuracy of gridded elevation models and for mapping uncertainty in digital terrain analysis. Progress in Physical Geography, v. 35, n. 6, p. 739764, 2011.

HAYAKAWA, E.H.; ROSSETTI, D.F.; VALERIANO, M.M. Applying DEM-SRTM for reconstructing a late Quaternary paleodrainage in Amazonia. Earth and Planetary Science
Letters, v. 297, p. 262-270, 2008.

HORTON, B.K.; DECELLES, P.G. The modern foreland basin system adjacent to the Central Andes. Geology, v. 25, n.10, p. 895-898, 1997.

HORTON, B.; DECELLES, P. Modern and ancient fluvial megafans in the foreland basin system of the central Andes, southern Bolivia: implications for drainage network evolution in fold-thrust belts. Basin Research, v. 13, p. 43-63, 2001.

ITT Visual Information Solution. ENVI (Environment for Visualizing Images). Pearl East Circle Boulder, CO. 2008.

JARVIS, A.; REUTER, H.I.; NELSON, A.; GUEVARA, E. Hole-filled SRTM for the globe Version 4, available from the CGIAR-CSI SRTM 90m Database: http://srtm.csi.cgiar. org, 2008.

JUNK, W. J.; CUNHA, C. N.; WANTZEN, K. M.; PETERMANN, P.; STRÜSSMANN, C.; MARQUES,M. I.; ADIS, J. Biodiversity and its conservation in the Pantanal of Mato Grosso, Brazil. Aquatic Sciences, v. 68, p. 278-309, 2006.

JUNK, W.J.; BAYLEY, P.B.; SPARKS, R.E. The flood pulse concept in river floodplain systems. Canadian Spec Publ Fish Aquatic Science, v. 106, p. 110-127, 1989.

KIRKBY, M. Modelling the links between vegetation and landforms. Geomorphology, v. 13, n. 1-4, p. 319-335, 1995.

KOUTSIAS, N.; KARTERISM, M.; CHUVIECO, E. The Use of Intensity-Hue-Saturation Transformation of Landsat-5 Thematic Mapper Data for Burned Land Mapping . Photogrammetric Engineering \& Remote Sensing, v. 66, n. 7, p. 829-839, 2000.

KUERTEN, S.; ASSINE, M.L. O rio Paraguai no megaleque do Nabileque, sudoeste do Pantanal Mato-Grossense, MS. Revista Brasileira de Geociências, v. 41, n. 4, p. 655-666, 2011.

LALONDE, T.; MESSINA, J.; SHORTRIDGE, A. The influence of land cover on shuttle radar topography mission (SRTM) elevations in low-relief areas. Transactions in GIS, v. 14, p. 461-479, 2010.

LATRUBESSE E.; AMSLER, M.; MORAIS, R.; AQUINO, S. The Geomorphologic response of a large pristine alluvial river to tremendous deforestation in the South American tropics: the case of the Araguaia River. Geomorphology, v. 113, p. 239$252,2009$.

LATRUBESSE, E.M.; COZZUOL, M.; RIGSBY, C.; SILVA, S.A.; ABSY, M.L.; JARAMILLO, C. The Late Miocene paleogeography of the Amazon basin and the evolution of the Amazon River. Earth-Science Reviews, v. 99, p. 99-124, 2010. 
LATRUBESSE, E. M.; FRANZINELLI, E. The late Quaternary evolution of the Negro River, Amazon, Brazil: implications for island and floodplain formation in large anabranching tropical systems. Geomorphology, v. 70, n. 3-4, p. 372-397, 2005.

MACEDO, H. A.; ASSINE, M. L.; SILVA, A.; PUPIM, F. N.; MERINO, E. R.; STEVAUX, J. C. Mudanças paleo-hidrológicas na planície do rio Paraguai, Quaternário do Pantanal. Revista Brasileira de Geomorfologia, v.15, n.1, p-71-81, 2014.

MANTELLI, L.R.; ROSSETTI, D.F.; ALBUQUERQUE, P.G.; VALERIANO, M.M. Applying SRTM digital elevation model to unravel Quaternary drainage in forested areas of Northeastern Amazonia. Computers \& Geosciences, v. 35, p. 2331-2337, 2009.

MARTINS, P.T.A.; GRADELLA, F.S.; CORRADINI, F.A.; PUPIM, F.N.; COELHO, J.O.M. Análise comparativa entre fusão de imagens CCD/HRC e TM/HRC na região dos rios Miranda e Abobral, Pantanal Sul-Mato-Grossense. Geografia (Rio Claro. Impresso), v. 34, p. 807-816, 2009.

MATHER, P.M. Is there any sense in remote sensing? Progress in Physical Geography, v. 34, n. 6, p. 739-740, 2010.

MDA FEDERAL. 2004. Landsat GeoCover ETM+ 2000 Edition Mosaics Tile N-03-05.ETM-EarthSat-MrSID, 1.0. USGS, Sioux Falls, South Dakota, 2000.

MENDES, D.; ASSINE, M.L.; PERROTTA, M.M.; CORRADINI, F.A.; SILVA, A. Application of fusion techniques to (ETM+/SRTM) images to recognize and interpret depositional landforms: an example from the Negro River alluvial plain, Pantanal Mato-Grossense. Geografia (Rio Claro. Impresso), v. 36, p. 85-95, 2011.

MERINO, E.R.; ASSINE, M.L.; PUPIM, F.N. Estilos fluviais e evidências de mudanças ambientais na planície do rio Miranda, Pantanal. Revista Brasileira de Geomorfologia, v. 14, n. 2, p. 127-134, 2013.

NAPIERALSKI, J.; HARBOR, J.; LI, Y. Glacial geomorphology and geographic information systems. Earth-Science Reviews, v. 85 , n. 1-2, p. 1-22, 2007.

OSTERKAMP, W.R.; HUPP, C.R. Fluvial processes and vegetation: glimpses of the past, the present, and perhaps the future. Geomorphology, v. 116, p. 275-285, 2010.

PAZ, A.R.D.; COLLISCHONN, W.; TUCCI, C.E.; PADOVANI, C.R. Large-scale modelling of channel flow and floodplain inundation dynamics and its application to the Pantanal (Brazil). Hydrological Processes, v. 25, n. 9, p. 1498-1516, 2011.

POTT, A.; POTT, V.J. Features and conservation of the Brazilian
Pantanal wetland. Wetlands Ecology and Management, v. 12, p. 547-552, 2004.

PRANCE, G.T.; SCHALLER, G.B. Preliminary Study of Some Vegetation Types of the Pantanal, Mato Grosso, Brazil. Brittonia, v. 34, n. 2, p. 228-251, 1982.

PUPIM, F.N.; ASSINE, M.L.; MERINO, E.R.; MACEDO, H.A.; SILVA, A. A planície interleques do rio Piquiri, bacia do Pantanal. Anais $\mathbf{5}^{\mathbf{0}}$ Simpósio de Geotecnologias no Pantanal, Campo Grande, MS, 2014. Embrapa Informática Agropecuária/ INPE, p. 848-857, 2014.

RABUS, B.; EINEDER, B.; ROTH, A.; BAMLER, R. The shuttle radar topography mission - a new class of digital elevation models acquired by spaceborne radar. ISPRS Journal of Photogrammetry \& Remote Sensing, v. 57, p. 241- 262, 2003.

ROSSETTI, D.F.; VALERIANO, M.M. Evolution of the lowest amazon basin modeled from the integration of geological and SRTM topographic data. CATENA, v. 70, n. 2, p. 253-265, 2007.

ROSSETTI, D.F. Multiple remote sensing techniques as a tool for reconstructing late Quaternary drainage in the Amazon lowland. Earth Surface Processes and Landforms, v. 35, p. 1234-1239, 2010.

ROSSETTI, D.F.; ZANI, H.; Cohen, M.L.; CREMON, É.H. A Late Pleistocene-Holocene wetland megafan in the Brazilian Amazonia. Sedimentary Geology, v. 281, p. 50-68, 2012.

SAADAT, H.; BONNELL, R.; SHARIFI, F.; MEHUYS, G.; NAMDAR, M.; ALE-EBRAHIM, S. Landform classification from a digital elevation model and satellite imagery. Geomorphology, v. 100, n. 3-4, p. 453-464, 2008.

SIART, C.; EITEL, B.; PANAGIOTOPOULOS, D. Investigation of past archaeological landscapes using remote sensing and GIS: a multi-method case study from Mount Ida, Crete. Journal of Archaeological Science, v. 35, n. 11, p. 2918-2926, 2008.

SIART, C.; BUBENZER, O.; EITEL, B. Combining digital elevation data (SRTM/ASTER), high resolution satellite imagery (Quickbird) and GIS for geomorphological mapping: A multicomponent case study on Mediterranean karst in Central Crete. Geomorphology, v. 112, n. 1-2, p. 106-121, 2009.

SILVA, A.; ASSINE, M.L.; ZANI, H.; SOUZA FILHO, E.E.; ARAÚJO, B.C. Compartimentação Geomorfológica do rio Paraguai na borda norte do Pantanal Mato-Grossense, Região de Cáceres - MT. Revista Brasileira de Cartografia, v. 59, n. 01, p. 73-81. 2007.

SILVA, J. S.V.; ABDON, M.M. Delimitação do Pantanal 
brasileiro e suas sub-regiões. Pesquisa Agropecuária Brasileira, v. 33, p. 1703-1711, 1998.

SMITH, M. J.; CLARK, C. D. Methods for the visualization of digital elevation models for landform mapping. Earth Surface Processes and Landforms, v. 30, n. 7, p. 885-900, 2005.

SMITH, M. J.; PAIN, C. F. Applications of remote sensing in geomorphology. Progress in Physical Geography, v. 33, n. 4, p. $568-582,2009$.

STEVAUX, J. C. The upper Paraná River (Brazil): Geomorphology, sedimentology and Paleoclimatology. Quaternary International, Gra-Bretanha, v. 21, p. 143-161, 1994.

STEVAUX, J. C.; SOUZA, I. A. Floodplain construction in an anastomosed river. Quaternary International, Amsterdam, v. 114, p. 55-66, 2004.

SINHA, R. Channel avulsion and floodplain structure in the Gandak-Kosi Interfan, north Bihar Plains, India. Zeitschriftfuer Geomorphologie: ementband, v. 103, p. 249-268, 1996.

SOARES, A. P., SOARES, P. C., ASSINE, M. L. Areiais e lagoas do Pantanal, Brasil: herança paleoclimática? Revista Brasileira de Geociências, v. 33, n. 2, p. 211- 224, 2003.

STOTT T. Review of research in fluvial geomorphology for the years 2006 and 2007. Progress in Physical Geography, v. 34, n. 2, p. 221-245, 2010.

STOTT, T. Fluvial geomorphology 2008-2009. Progress in Physical Geography, v. 35, n. 6, p. 810-830, 2011.

SYVITSKI, J.P.M; OVEREEM, I.; BRAKENRIDGE, G.R.; HANNON M. Floods, floodplains, delta plains - A satellite imaging approach. Sedimentary Geology, v. 267-268, p. 1-14, 2012.

TINÓS, T. M.; FERREIRA, M.V.; RIEDEL,P.S.; ZAINE, J.E. Aplicação e Avaliação de Metodologia de Classificação Automática de Padrões de Formas Semelhantes de Relevo. Revista Brasileira de Geomorfologia, v. 15, n. 3, p. 353 - 370, 2014.

TRICART, J. El Pantanal: un ejemplo del impacto geomorfologico sobre El ambiente. Informaciones Geograficas (Chile), v. 29, p. 81-97, 1982.

TU, T.M.; SU, S.C.; SHYU, H.C.; HUANG, P.S. Efficient intensity-hue-saturation based image fusion with saturation compensation. Optical Engineering, v. 40(5), p. 720-728, 2001.

USSAMI, N.; SHIRAIWA, S.; DOMINGUEZ, J.M.L. Basement reactivation in a sub-Andean foreland flexural bulge: Pantanal wetland, SW Brazil. Tectonics, v.18, n. 1, 25-39, 1999.

VALENTE, C.; LATRUBESSE, E. The Quaternary record of the largest intra-cratonic basin of South America and its paleohydrological significance for the tropics: the Bananal basin, Brazil. Paleogeography, Palaeoclimatology, Palaeoecology, v. 356-357, p. 62-74, 2012.

VALERIANO, M.M.; KUPLICH, T.M.; STORINO, M.; AMARAL, B.D.; MENDES, J.N.; LIMA, D.J. Modeling small watersheds in Brazilian Amazonia with shuttle radar topographic mission-90 m data. Computers \& Geosciences, v. 32, p. 1169-1181, 2006.

VERSTAPPEN, H. T. Remote sensing in geomorphology. Elsevier Scientific Publishing Company, 1977.

VITEK, J. D.; GIARDINO, J. R.; FITZGERALD, J. W. Mapping geomorphology: A journey from paper maps, through computer mapping to GIS and Virtual Reality. Geomorphology, v. 16, n. 3, p. 233-249, 1996.

WALSH, S. J.; BUTLER, D. R.; MALANSON, G. P. An overview of scale, pattern, process relationships in geomorphology: a remote sensing and GIS perspective. Geomorphology, v. 21, n. 3-4, p. 183-205, 1998.

YANG, L.; MENG, X.; ZHANG, X. SRTM DEM and its application advances. International Journal of Remote Sensing, v. 32, n. 14, p. 3875-3896, 2011.

ZANI, H.; ASSINE, M. L.; SILVA, A.; CORRADINI, F. A. Redes de drenagem distributária e formas deposicionais no Megaleque do Taquari, Pantanal: uma análise baseada no MDE-SRTM. Revista Brasileira de Geomorfologia, v. 10, p. 21-28, 2009.

ZANI, H.; ASSINE, M.L.; MCGLUE, M.M. Remote sensing analysis of depositional landforms in alluvial settings: Method development and application to the Taquari megafan, Pantanal (Brazil). Geomorphology, v. 161, p. 82-92, 2012. 